\title{
ON THE AUTOMORPHISM GROUP OF A LINEAR ALGEBRAIC MONOID
}

\begin{abstract}
MOHAN S. PUTCHA
AbSTRACT. Let $S$ be a connected regular monoid with zero. It is shown that an automorphism of $S$ is inner if and only if it sends each idempotent of $S$ to a conjugate idempotent. In the language of semigroup theory, the automorphism group of $S$ maps homomorphically into the automorphism group of the finite lattice of $\mathcal{G}$-classes of $S$, and the kernel of this homomorphism is the group of inner automorphisms of $S$. In particular, if the $\mathcal{K}$-classes of $S$ are linearly ordered, then every automorphism of $S$ is inner.
\end{abstract}

Throughout this paper $Z^{+}$will denote the set of all positive integers and $K$ an algebraically closed field. $\Re_{n}(K)$ denotes the monoid of all $n \times n$ matrices over $K$. $\mathrm{GL}(n, K)$ denotes the group of units of $\mathfrak{N}_{n}(K)$, and $\operatorname{SL}(n, K)$ the group of matrices of determinant 1 in $\mathfrak{N}_{n}(K)$. We will follow the notation and terminology of $[2,4]$ concerning linear algebraic monoids. Let $S$ be a connected algebraic monoid with group of units $G$. By an automorphism of $S$ is meant a semigroup automorphism $\sigma$ of $S$ such that both $\sigma$ and $\sigma^{-1}$ are polynomial maps. An automorphism $\sigma$ of $S$ is inner if there exists $g \in G$ such that $\sigma(a)=g^{-1} a g$ for all $a \in S$. We let $\mathcal{Q}(S)$ denote the finite lattice of all regular $q$-classes of $S$, and $E(S)$ the partially ordered set of all idempotents of $S$. It follows from the work of the author $[4,5]$ and Renner $[\mathbf{7 , 8}]$ that $S$ is regular if and only if the closure of the radical of $G$ is a Clifford semigroup. In particular, if $S$ has a zero then $S$ is regular if and only if $G$ is a reductive group.

THEOREM 1. Let $S$ be a connected regular monoid with zero and $\sigma$ an automorphism of $S$. Then $\sigma$ is an inner automorphism of $S$ if and only if $\sigma(J)=J$ for all $J \in \mathcal{U}(S)$ (i.e. $\sigma(e)$ is a conjugate of e for all $e \in E(S)$ ).

Proof. Suppose $\sigma(J)$ for all $J \in \mathcal{U}(S)$. We must show that $\sigma$ is inner. Let $G$ denote the group of units of $S$, and let $T$ be a maximal torus of $G$. Suppose first that $T=G$. Then $\sigma(e)=e$ for all $e \in E(\bar{T})$. We prove by induction on $\operatorname{dim} T$ that $\sigma$ is the identity map. First suppose that $\operatorname{dim} T=1$. Then by [1, Exercise 4, p. 57] either $\sigma(t)=t$ for all $t \in T$, or else $\sigma(t)=t^{-1}$ for all $t \in T$. In the latter case $t \sigma(t)=1$ for all $t \in T$ and, hence, for all $t \in \bar{T}$. Since $0 \in \bar{T}$, this is a contradiction. So let $\operatorname{dim} T>1$. Let $F=\{t \in T \mid \sigma(t)=t\}^{c}$. Let $e \in E(\bar{T}), e \neq 0$. Let

$$
T_{e}=\{a \in T \mid a e=e\}^{c} \text {. }
$$

Received by the editors September 20, 1982 and, in revised form, November 29, 1982.

1980 Mathematics Subject Classification. Primary 20M10; Secondary 20 G99.

Key words and phrases. Monoid, algebraic, automorphism, $\mathcal{G}$-class.

${ }^{1}$ This research was partially supported by NSF Grant MCS8025597.

(c) 1983 American Mathematical Society $0002-9939 / 82 / 0000-1213 / \$ 01.75$ 
Since $\sigma(e)=e, \sigma\left(T_{e}\right)=T_{e}$. Since $e$ is the zero of $\bar{T}_{e}$, we see by the induction hypothesis that $T_{e} \subseteq F$. Thus $e \in \bar{F}$. So $E(\bar{T}) \backslash\{0\} \subseteq \bar{F}$. There exists $f \in E(\bar{T})$, $f \neq 1,0$. So [2, Theorem 1.4] there exists $h \in E(\bar{T}), h \neq 0$, such that $f h=0$. Since $f$, $h \in \bar{F}, 0 \in \bar{F}$. So $E(\bar{T})=E(\bar{F})$. By [2, Theorem 1.4], $\operatorname{dim} T=\operatorname{dim} F$. Thus $T=F$ and $\sigma$ is the identity map. In particular, the automorphism group of $\bar{T}$ is isomorphic to a subgroup of the group of automorphisms of $E(\bar{T})$ and, hence, is a finite group.

Let us now consider the general case. Since all maximal tori of $G$ are conjugate, we can assume without loss of generality that $\sigma(T)=T$. Let $\Lambda \subseteq E(\bar{T})$ be a crosssection lattice of $S$ (see [6]). In other words, if $e, f \in \Lambda, f \in S e S$, then $e \geqslant f$, each idempotent of $S$ is conjugate to an idempotent in $\Lambda$, and no two idempotents in $\Lambda$ are conjugate. So $\sigma(\Lambda) \subseteq E(\bar{T})$ is also a cross-secton lattice of $S$. By [6, Theorem 11] there exists $u \in W$, the Weyl group of $G$ relative to $T$, such that $\sigma(\Lambda)=u^{-1} \Lambda u$. Thus, without loss of generality, we can assume that $\sigma(\Lambda)=\Lambda$. Let $e \in \Lambda$. Then $e$, $\sigma(e) \in \Lambda$. By hypothesis, $e q \sigma(e)$. Since $\Lambda$ is a cross-section lattice of $S, e=\sigma(e)$. So $\sigma(e)=e$ for all $e \in \Lambda$. Let $F=\{t \in T \mid \sigma(t)=t\}^{c}$. Since the automorphism group of $\bar{T}$ is finite, we see that there exists $k \in Z^{+}$such that $\sigma^{k}(a)=a$ for all $a \in \bar{T}$. So by [3, Lemma 1.13], $\Lambda \subseteq \bar{F}$. Since $\Lambda$ contains a maximal chain of $E(\bar{T})$, we see by [2, Theorem 1.4] that $\operatorname{dim} F=\operatorname{dim} T$, so $T=F$. Thus $\sigma(t)=t$ for all $t \in T$. Let $\Gamma$ be a maximal chain in $E(\bar{T})$, and let $B=\{a \in G \mid a e=e a e$ for all $e \in \Gamma\}$. Since $\sigma(\Gamma)=\Gamma$, we see that $\sigma(B)=B$. Since $G$ is a reductive group, we see by [4, Theorem 4.5] that $B$ is a Borel subgroup of $G$. Clearly $T \subseteq B$. We see by [1, Theorem 27.4(b) and 9, Theorem 11.4.3] that $\sigma$ is an inner automorphism.

ExAmple. Let $S$ denote the Zariski closure in $\Re_{3}(K) \times \Re_{3}(K)$ of $\left\{\left(\alpha A, \alpha\left(A^{-1}\right)^{T}\right) \mid \alpha \in K, A \in \mathrm{SL}(3, K)\right\}$. Then $S$ is a connected regular monoid with zero, $\mathcal{Q}(S)=\left\{G, J, J_{1}, J_{2}, 0\right\}$ with $G>J>J_{i}>0, i=1,2$. Let $e, e_{1}, e_{2}$, denote diagonal matrices with the respective diagonals being $((1,0,0),(0,0,1)),((1,0,0)$, $(0,0,0)),((0,0,0),(0,0,1))$. Then $e \in J, e_{1} \in J_{1}, e_{2} \in J_{2}$ and $\left\{1, e, e_{1}, e_{2}, 0\right\}$ is a cross-section lattice of $S$. Let $\sigma: S \rightarrow S$ be given by $\sigma(A, B)=(B, A)$. Then $\sigma$ is an automorphism of $S$ which is not inner. Note that $\sigma$ induces a nontrivial automorphism of $थ(S): \sigma(G)=G, \sigma(J)=J, \sigma(0)=0, \sigma\left(J_{1}\right)=J_{2}, \sigma\left(J_{2}\right)=J_{1}$.

Theorem 1 can be restated as follows.

THEOREM 2. Let $S$ be a connected regular monoid with zero. Then the automorphism group of $S$ maps homomorphically into the automophism group of $\mathcal{U}(S)$. The kernel of this homomorphism is the group of inner automorphisms of $S$.

COROLlaRY. Let $S$ be a connected regular monoid with zero such that the G-classes of $S$ are linearly ordered. Then every automorphism of $S$ is inner.

REMARK. The above corollary applies to the multiplicative monoid $\mathfrak{R}_{n}(K)$. Note that the map $A \rightarrow\left(A^{-1}\right)^{T}$ is an automorphism of $\operatorname{GL}(n, K)$ which is not inner.

\section{REFERENCES}

1. J. E. Humphreys, Linear algebraic groups, Springer-Verlag, Berlin and New York, 1981.

2. M. S. Putcha, Linear algebraic semigroups, Semigroup Forum 22 (1981), 287-309.

3 . , Connected algebraic monoids, Trans. Amer. Math. Soc. 272 (1982), 693-709. 
4. __ A semigroup approach to linear algebraic groups, J. Algebra (to appear).

5. __ Reductive groups and regular semigroups, J. Algebra (submitted).

6. Idempotent cross-sections of $\mathcal{G}$-classes, Semigroup Forum (to appear).

7. L. Renner, Algebraic monoids, Ph. D. Thesis, Univ. of British Columbia, 1982.

8. __ Reductive monoids are von-Neumann regular (to appear).

9. T. A. Springer, Linear algebraic groups, Birkhaüser, Basel, 1981.

Department of Mathematics, North Carolina State University, Raleigh, North Carolina 27650 